\title{
Revista
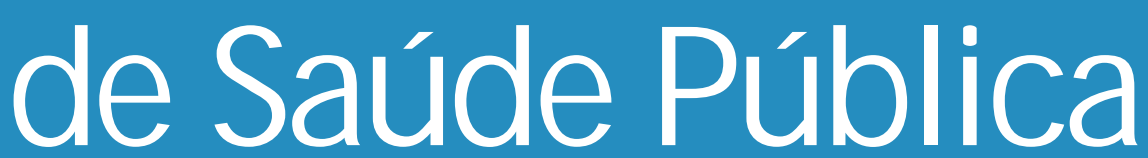

$\begin{array}{llllll}\mathbf{O} & \mathbf{U} & \mathbf{R} & \mathbf{N} & \mathbf{A} & \mathbf{L}\end{array}$

0 F

$\mathbf{P} \mathbf{U}$

B L I C

H E A L T H

\section{G astroenterites e infecções respiratórias agudas em crianças menores de 5 anos, em área da região Sudeste do Brasil, 1986-1987. II - Diarréias}

\section{Gastroenteritis and acute respiratory infections among children up to 5 years old in an area of Southeastern Brazil, 1986-1987. II - Diarrhea}

Eliseu Alves Waldman, Rita de Cassia Barradas Barata, José Cássio de Moraes, lone Aquemi G uibu e Maria do Carmo S. T. Timenetsky

Departamento de Epidemiologia da Faculdade de Saúde Pública da U niversidade de São Paulo. São Paulo, SP - Brasil (E.A.W.); Departamento de M edicina Social da Faculdade de Ciências M édicas da Santa Casa de M isericórdia de São Paulo. São Paulo, SP - Brasil (R.C.B.B., J.C.M., I.A.G.); Instituto Adolfo Lutz. São Paulo, SP - Brasil (M.C.S.T.T.) área da região Sudeste do Brasil, 1986-1987. II - Diarréias. Rev. Saúde Pública, 31 (1): 62-70, 1997. 


\title{
G astroenterites e infecções respiratórias agudas em crianças menores de 5 anos, em área da região Sudeste do Brasil, 1986-1987. II - Diarréias*
}

\section{G astroenteritis and acute respiratory infections among children up to 5 years old in an area of Southeastern Brazil, 1986-1987. II - D iarrhea}

\author{
Eliseu Alves Waldman, Rita de Cassia Barradas Barata, José Cássio de Moraes, lone Aquemi \\ G uibu e Maria do Carmo S. T. Timenetsky
}

Departamento de Epidemiologia da Faculdade de Saúde Pública da Universidade de São Paulo. São Paulo, SP - Brasil (E.A.W.); D epartamento de Medicina Social da Faculdade de Ciências M édicas da Santa Casa de Misericórdia de São Paulo. São Paulo, SP - Brasil (R.C.B.B., J.C.M., I.A.G.); Instituto Adolfo Lutz. São Paulo, SP - Brasil (M.C.S.T.T.)

\begin{abstract}
Resumo
Introdução

O declínio da morbi-mortalidade pelas gastroenterites é, em boa parte, responsável pela queda da mortalidade infantil e da mortalidade por doenças infecciosas nos países do terceiro mundo. Esse agravo ainda se destaca, nesses países, como importante problema de saúde pública, especialmente, entre os menores de 5 anos.
\end{abstract}

Objetivos

Descrever aspectos do comportamento das gastroenterites entre crianças menores de 5 anos, residentes em 5 bairros do Município de São Paulo.

Material e Método

Estudou-se uma amostra probabilística $(\mathrm{N}=468)$ de crianças menores de 5 anos, residentes em 5 áreas do Município de São Paulo, SP (Brasil), acompanhada durante um ano, por meio de entrevistas mensais.

Resultados

\begin{abstract}
Durante o acompanhamento foram identificados 139 episódios de diarréia, com uma duração média de 5,5 dias, $10 \%$ dos casos prolongaram-se por 15 dias ou mais. Em $20 \%$ dos episódios havia ao menos outra pessoa na família com diarréia. A incidência foi de 2,78 casos por 100 crianças/mês, sendo mais elevada nos menores de 2 anos. Em 46,1\% dos episódios de gastroenterite as crianças não demandaram assistência médica tendo sido tratadas pelas próprias mães, ou não receberam qualquer tratamento; em 51,8\% dos episódios o atendimento foi feito em serviços de assistência primária à saúde e somente $2,1 \%$ dos casos necessitaram tratamento hospitalar. Nenhuma criança evoluiu para óbito. Entre as medidas terapêuticas mais utilizadas estão a reidratação oral $(25,2 \%)$ e a antibioticoterapia associada à reidratação oral $(11,5 \%)$; em somente 2 casos foi feita reidratação endovenosa. Alguns fatores socioeconômicos e antecedentes pessoais mostraram-se associados à ocorrência de diarréias, entre eles, as condições da habitação, saneamento básico e renda familiar "per capita" e história pregressa de diarréias freqüentes.
\end{abstract}

\footnotetext{
* Pesquisa financiada pelo Conselho Nacional de Desenvolvimento Científico e Tecnológico/CNPq. Processo 40.0890/85 e pela Financiadora de Estudos e Projetos/FINEP. Processo 43.85.01.01.00.

Correspondência para/Correspondence to: Eliseu Alves Waldman - Departamento de Epidemiologia da Faculdade de Saúde Pública da Universidade de São Paulo. Av. Dr. Arnaldo, 715 - 01246-904 São Paulo, SP - Brasil. E-mail: eawaldman@usp.br

Edição subvencionada pela FAPESP. Processo 96/5999-9.

Recebido em 20.3.1995. Reapresentado em 31.3.1996. Aprovado em 24.5.1996.
} 
Discussão

Objectives

\section{Material and Method}

Results

Discussion
Os resultados obtidos parecem refletir a tendência de diminuição da morbimortalidade por diarréias no Município de São Paulo, durante a década de 80, período em que houve acentuada queda nas internações hospitalares por essa causa. Tal tendência deve ser acompanhada atentamente, pois influenciará modificações nas características da demanda de assistência à saúde infantil.

\section{Gastroenterite, epidemiologia. Fatores de risco.}

\section{Abstract}

The decrease morbi-mortality gastroenteritis in is related to the factor responsible largely for the fall in infant mortality and mortality from communicable diseases in developing countries. Nevertheless, diarrhea is still a considerable public health problem in these countries, especially among children under 5 years old.

To describe some aspects of the of gastroenteritis epidemiology among children up to 5 years old, resident in areas of $S$. Paulo county.

A probabilistic sample of children up to 5 years old was studied $(n=468)$. The epidemiological survey was undertaken in five areas $S$. Paulo county (Brazil) from March 1986 to May 1987. Data were obtained through household interviews once a month over a year.

During the follow-up 139 diarrhea episodes were registered, with a mean duration of 5.5 days. Twenty percent of the diarrhea events were followed by at least one other case in the household. The incidence of gastroenteritis was 2.78 episodes per 100 children/month. The highest incidence affected the children of up to 2 years of age. In $46.1 \%$ of the gastroenteritis episodes medical assistance was not sought, the children were treated by their mothers or not at all; $51.8 \%$ of the diarrhea events were attended to by the primary health care service, and only $2.1 \%$ were attended to by a hospital. No child died as a consequence of diarrhea. Of the therapeutical interventions used the most frequent were oral rehydration $(25.2 \%)$ and oral rehydration with antibiotics $(11.5 \%)$. Various socio-economic and personal background factors such as living conditions, water supply, sewarage, coverage; family income per capita and prior history of frequent gastroenteritis were associated with a higher incidence of gastroenteritis.

The results seen to reflect the tendency of morbi-mortality by gastroenteritis to decrease in S. Paulo county during the 1980's when hospital treatment of diarrhea presented a considerable reduction. This tendency must be observed closely, because it will influence the changes to be mode in the kind of demand for medical care.

\section{G astroenteritis, epidemiology. Risk factors.}

\section{INTRO DUÇÃO}

Assiste-se, nas últimas décadas, importantes alterações nos padrões de morbi-mortalidade em todos os continentes. Embora este processo tenha obedecido a velocidades distintas conforme a área analisada, suas repercussões nos indicadores demográficos e sanitários foram profundas cabendo citar o declínio da mortalidade infantil e da mortalidade por doenças infecciosas e o aumento médio na expecta- tiva de vida, que no período de 1950 a 1990, variou em torno de 10 anos nos países desenvolvidos e de 21 nos do terceiro mundo ${ }^{23}$.

Analisando este fenômeno no âmbito da América Latina, verifica-se queda importante das taxas de mortalidade infantil principalmente às custas das doenças infecciosas e parasitárias e um ganho expressivo na expectativa de vida, elevando-se, durante o período de 1930 a 1980 , de 40 para 64 anos $^{4}$. Esta evolução favorável não impede a manutenção 
de uma grande heterogeneidade na evolução desses indicadores no continente latino-americano. Se forem tomados os coeficientes de mortalidade infantil dessa região, relativos ao ano de 1993, observa-se que os mesmos variavam de 9/1.000 nascidos vivos a 85/1.000 nascidos vivos, respectivamente, em Cuba e no Haiti ${ }^{11}$.

Apesar da evolução desses indicadores nos países do terceiro mundo estar em boa parte relacionada ao importante declínio da morbi-mortalidade pelas gastroenterites, este agravo ainda se destaca, nesses países, entre os principais problemas de saúde pública atingindo especialmente os menores de 5 anos. Em algumas regiões subdesenvolvidas a doença diarréica ainda é responsável por mais vidas perdidas do que a soma de todas as outras causas de morte ${ }^{14}$.

A despeito de sua importância, as estimativas da incidência das gastroenterites não são facilmente disponíveis, e quando existentes, devem ser analisadas com cautela, pois podem variar muito de país para país, conforme a definição de caso adotada e a sensibilidade dos sistemas de informação disponíveis ${ }^{27}$. Mesmo com essa ressalva, mas com o objetivo de situar a magnitude desse agravo, vale citar estimativas referentes a 1985 que estabelecem em cerca de 3 bilhões o número de episódios de diarréia, em todo mundo, com aproximadamente 5 milhões de óbitos, dos quais $80 \%$ atingindo menores de 5 anos ${ }^{19}$.

Pela dificuldade de se obter dados de morbidade, de base populacional, relativos às gastroenterites, face à exigüidade de registros sistemáticos disponíveis, boa parte das informações relativas a esse agravo, em menores de 5 anos, origina-se de estudos de demanda de serviços de saúde, ou de amostras de determinados grupos populacionais, dificultando assim a obtenção de valores estimados que possam ser generalizados para populações mais amplas ${ }^{2}, 9,10,15,16,18$.

No Brasil são poucos os estudos longitudinais realizados para obtenção de informações relativas ao comportamento das diarréias, entre eles vale citar os realizados em comunidades rurais da região nordeste $^{13}$, periurbanas de Manaus ${ }^{12}$ e urbana de Pelotas $^{28}$. No Estado de São Paulo existem informações semelhantes provenientes de alguns inquéritos domiciliares como os realizados por Carvalheiro ${ }^{8}$, em Ribeirão Preto, e Benício e col. ${ }^{5}$, no Município de São Paulo. Essa exigüidade de informações no País a respeito do espectro das manifestações clínicas das gastroenterites, das condutas terapêuticas, da demanda de serviços gerada por esse agravo e de seu comportamento epidemiológico, dificulta a elaboração de programas de controle fundamentados no conhecimento da estrutura epidemiológica do País.

O presente artigo mostrará os resultados do acompanhamento de uma coorte de crianças menores de 5 anos, residentes em 5 bairros do Município de São Paulo, com o objetivo de descrever aspectos das condições de vida das crianças estudadas, como habitação e acesso a serviços de infra-estrutura urbana, assim como descrever aspectos do comportamento das gastroenterites nessa população, especialmente, duração, gravidade, evolução, tipo de atendimento e conduta terapêutica nos episódios registrados. Será também estimada a frequiência e distribuição das diarréias na população estudada, especialmente, a incidência, diferenciais por faixa etária e por área de residência e sazonalidade.

\section{MATERIAL E MÉTO DO}

Foram estudadas 468 crianças do Município de São Paulo, selecionadas por uma amostra probabilística, entre os menores de 5 anos residentes no subdistrito de paz da Barra Funda, nos bairros de Vila Ramos e Itaberaba, pertencentes ao subdistrito de paz da Brasilândia, e de Vila Penteado e Parque Tietê situados no subdistrito de paz de Nossa Senhora do Ó. A escolha dos bairros foi feita com base em indicadores demográficos, econômicos e sanitários obtidos em órgãos oficiais*, procurando selecionar populações com condições de vida diversificadas de modo a incluir crianças submetidas a diferentes estruturas epidemiológicas e diferentes fatores de risco para gastroenterites.

Cada criança foi acompanhada mensalmente durante 12 meses através de visitas domiciliares realizadas por entrevistadores com treinamento em inquéritos domiciliares. A descrição detalhada dos procedimentos para determinação da amostra e levantamento dos dados, assim como a caracterização das crianças e de suas famílias encontramse publicados no primeiro artigo desta série (Barata e col. ${ }^{3}$ ).

Definiu-se caso de diarréia a ocorrência de três ou mais evacuações líquidas em 24 horas, com ou sem sangue, pus ou muco. O reaparecimento dos sintomas após 7 dias, ou mais, foi considerado como novo episódio.

A caracterização do Município de São Paulo foi feita com fundamento em dados e indicadores obtidos nas Fundações SEADE e IBGE e em publicações especializadas. Os dados levantados pelo acompanhamento das 468 crianças foram processados em microcomputador mediante a utilização do "software" Epi Info (CDC), versão nº 6. Na análise estatística para testes de associação foram utilizados o teste "t" para comparação entre médias e o qui-quadrado. Para variáveis que apresentaram associação com a ocorrência de gastroenterites, foram calculados os riscos relativos e seus intervalos de confiança.

\footnotetext{
* Dados fornecidos pela Fundação SEADE - São Paulo.
} 


\section{RESULTADOS}

\section{Características do Município de São Paulo e da Área Estudada}

Ao serem tomados alguns indicadores relativos a evolução das condições de vida da população residente no Município de São Paulo, dos últimos 20 anos, verifica-se uma evolução favorável. Entre eles está o saneamento básico que apresentou importante incremento a partir da segunda metade da década de 70. A cobertura da rede de água e esgoto ampliou-se, respectivamente, de $59 \%$ e $35 \%$, em 1975 , para $96 \%$ e $60 \%$, em $1985^{21}$. No período de 1974 a 1984 houve também ampliação do acesso da população aos serviços de saúde, com aumento do número de unidades sanitárias no município de, aproximadamente, $50 \%$, enquanto que o número de médicos por habitante praticamente triplicou ${ }^{21}$. Dados oficiais sugerem que a partir de 1988 todas as crianças de 7 a 14 anos poderiam ter acesso às escolas de primeiro grau, e que de 1980 a 1988 houve um aumento de $20 \%$ nas matrículas em escolas de segundo grau*.

Após um exacerbado período de crescimento populacional a partir de meados deste século, às custas principalmente de correntes migratórias, quando tivemos, por exemplo, taxas de crescimento decenal da população do município nas décadas de 60 e 70 em torno de $59,7 \%$ e $43,3 \%$, respectivamente, verificou-se no período de 1980 a 1991 uma queda dessa taxa para 13,6\%** no correr dos 11 anos.

Foram verificadas alterações importantes ao se analisar os indicadores das condições de saúde da população residente no Município de São Paulo, relativos às três últimas décadas. Desde 1980, as doenças crônico-degenerativas situam-se entre as três principais causas de óbito, ficando em quarto lugar a pneumonia. A mortalidade infantil que havia apresentado aumento em cerca de $30 \%$ na década de 60, atingindo 94,4/1.000 nascidos vivos, em 1973, declina, em seguida, acentuadamente até situar-se em 25,6/1.000 nascidos vivos em 1992. Essa queda importante da mortalidade infantil nas últimas duas décadas, abrangeu todos os estratos sociais, mantendo, porém, os diferenciais existentes. Se tomarmos o período de 1973 a 1983, a queda da mortalidade infantil entre as parcelas da população de nível socioeconômico mais elevado foi de 62,1 para $32,1 / 1.000$ nascidos vivos; nos seguimentos de renda intermediária, esse declínio foi de 83,1 para 37,8/
1.000 nascidos vivos; e, por fim, entre os mais pobres tivemos uma diminuição de 114,5 para $50,4 /$ 1.000 nascidos vivos ${ }^{21}$.

Analisando a distribuição proporcional da mortalidade infantil por causa, verificaram-se profundas modificações nas duas últimas décadas. Em 1971, as duas principais causas da mortalidade infantil, no Município de São Paulo, eram as diarréias e a pneumonia, contribuindo com $26,2 \%$ e $21,8 \%$ dos óbitos, respectivamente. Em 1992, a mortalidade infantil determinada por afecções originadas no período perinatal correspondia a cerca de $50,89 \%$ dos óbitos, enquanto que, as pneumonias e as diarréias situavam-se como a segunda e quarta principais causas, contribuindo, respectivamente, com $18,13 \%$ e $5,35 \%$ dos óbitos. O coeficiente de mortalidade infantil por doenças preveníveis por vacinação que ficava em torno de 1,4/1.000 nascidos vivos, em 1979, com quase 300 óbitos por sarampo, praticamente desaparece no início da década de 90.

Ao cotejarmos os indicadores demográficos, econômicos, sociais e de saúde apresentados na Tabela 1 , referentes ao Município de São Paulo e às regiões objeto da presente pesquisa, verifica-se por meio desses indicadores a existência de diferenciais que situam os subdistritos de paz da Barra Funda, N. S. do Ó e de Brasilândia, respectivamente, um pouco acima, em níveis semelhantes e um pouco abaixo daqueles encontrados no Município de São Paulo, como um todo.

\section{Condições de Habitação e de Acesso ao Saneamento Básico para as Famílias Estudadas}

As condições de habitação eram adequadas para $55,9 \%$ das famílias estudadas, $41,1 \%$ delas habitavam construções com condições inadequadas de espaço, ventilação e iluminação, e as demais (3\%) residiam em habitações precárias (barracos).

O número médio de pessoas por dormitório foi de 3,4 com mediana de 4. Em 15,8\% das famílias havia seis ou mais pessoas por dormitório. $\mathrm{O}$ número médio de pessoas por cama foi de $1,4 \mathrm{com}$ mediana igual a 1. Entretanto, em 2,7\% dos domicílios havia três ou mais pessoas por cama.

Dos domicílios estudados, $97,6 \%$ contavam com água encanada e solução adequada para o destino dos dejetos (rede de esgoto ou fossas sépticas). Apenas 2,4\% das famílias não dispunham de instalações sanitárias e de água encanada no domicílio.

*Prefeitura Municipal de São Paulo/ Secretaria de Saúde: Centro de Epidemiologia, Pesquisa e Informação ( CEPI ) - Dados inéditos

** Dados fornecidos pela Fundação IBGE - Brasil. 
Tabela 1 - Alguns indicadores demográficos e sociais das áreas estudadas e do M unicípio de São Paulo.

Table 1 - Some sociodemographics indicators of the studied areas and of the municipality of S. Paulo.

\begin{tabular}{|c|c|c|c|c|c|c|c|c|}
\hline Áreas & $\begin{array}{c}\% \text { de } \\
>\text { de } \\
50 \text { anos* }\end{array}$ & $\begin{array}{l}\text { Mort. } \\
\text { Infantil } \\
1993^{* *}\end{array}$ & $\begin{array}{c}\text { Mort. } \\
\text { Prop. } \\
<1 \text { ano \% } \\
1993^{* *}\end{array}$ & $\begin{array}{c}\% \text { de } \\
\text { Analfabetos* } \\
\%\end{array}$ & $\begin{array}{c}\% \text { da } \\
\text { população } \\
\text { com } \\
20 \text { grau } \\
\text { completo* }\end{array}$ & $\begin{array}{l}\text { Hab./ } \\
\text { dom.* }\end{array}$ & $\begin{array}{c}\% \text { de } \\
\text { chefes de } \\
\text { família } \\
\text { com renda } \\
\text { média de } \\
\text { até } 3 \text { SM* }\end{array}$ & $\begin{array}{c}\text { Renda } \\
\text { média } \\
\text { nominal } \\
\text { do chefe } \\
\text { da família } \\
\text { em SM* }\end{array}$ \\
\hline B. Funda & 23,4 & 22,2 & 3,7 & 11,8 & 45,1 & 2,9 & 30,2 & 7,0 \\
\hline N.S. do Ó & 16,0 & 23,3 & 6,3 & 18,5 & 20,9 & 3,7 & 40,3 & 5,3 \\
\hline Brasilândia & 10,7 & 32,0 & 12,9 & 25,0 & 10,6 & 4,1 & 47,5 & 3,6 \\
\hline Mun. de S. Paulo & 15,3 & 25,7 & 7,9 & 19,3 & 28,4 & 3,8 & 33,8 & 8,3 \\
\hline
\end{tabular}

SM : Salários-mínimos

* Censo Demográfico 1991 - Dados fornecidos pela Fundação IBGE, Brasil

** Dados fornecidos pela Fundação SEADE, SP

\section{Comportamento das Diarréias}

Ocorreram 139 episódios de gastroenterites agudas entre as 416,5 crianças/ano estudadas. A mediana da duração dos episódios foi de 3 dias, a média de 5,5 dias e o intervalo de confiança variou de 4,3 a 6,7 dias. Em 77\% dos episódios a duração foi inferior a 7 dias; em $10 \%$ variou entre 7 e 9 dias, e em 9,3\% dos casos prolongaram-se por 15 dias ou mais.

A duração dos episódios mostrou pequena variação de acordo com a sintomatologia apresentada. Nos casos em que o único sintoma foi a diarréia, o período médio de duração foi de 7,6 dias; nos casos com diarréia e vômitos esse período foi em média de 5,0 dias. Quando além de diarréia e vômitos havia febre, a duração média foi de 5,8 dias e nos casos com diarréia e febre ela chegou a 7,0 dias. A febre apareceu associada à diarréia em $28 \%$ dos casos e os vômitos em $22,3 \%$ deles. Para $42,4 \%$ dos episódios relatados, o único sinal registrado foi a diarréia. Em 5 episódios foi referida a presença de muco e sangue nas fezes e em um episódio houve a eliminação de Ascaris lumbricoides. O número médio de evacuações por dia foi de 4,5 com o intervalo de confiança variando entre 4,1 e 4,9 evacuações/dia. Em $23 \%$ dos casos foram referidas 6 ou mais evacuações/dia.

Em $80 \%$ dos episódios, a criança estudada foi o único membro da família a apresentar diarréia. Nos outros $20 \%$ em que além da criança estudada havia outra pessoa com diarréia, verificou-se, em média, cerca de 2,9 pessoas acometidas por família.

Em 32,4\% dos episódios não houve demanda a nenhum tipo de assistência; em $13,7 \%$ o atendimento foi feito por um leigo e em $51,8 \%$ o atendimento foi feito em serviços de assistência primária à saúde das redes pública e privada. Somente 3 casos $(2,1 \%)$ necessitaram tratamento hospitalar, nenhum deles evoluiu para óbito.
Relacionando os sintomas ao tipo de atendimento, verificou-se que nos casos que apresentaram apenas diarréia ou diarréia e febre, predominou a ausência de atendimento e o atendimento leigo. Nos casos de diarréia e vômito ou, ainda, diarréia, vômitos e febre predominou a busca de assistência nos serviços de saúde $(\mathrm{p}<0,01)$.

Quanto às condutas terapêuticas, em 32,4\% dos episódios não foi feito nenhum tratamento, enquanto que, em outros $21,6 \%$ o atendimento foi oferecido por leigo ou farmacêutico. Em 25,2\% dos episódios a terapêutica baseou-se na reidratação oral e em apenas 2 casos foi feita reidratação endovenosa. Em 11,5\% deles, além da reidratação oral, foram prescritos antibióticos. Nos demais casos foram utilizados exclusivamente orientação dietética ou, além dela, carbonato de cálcio ou outras drogas sintomáticas.

Para os episódios de diarréia e diarréia e vômitos predominou a conduta expectante seguindo-se a reidratação oral associada a uso de antibióticos. Nos casos de diarréia e febre predominou o uso dos reidratantes orais. Os episódios de diarréia com febre e vômitos foram tratados mais freqüentemente com reidratação oral e antibióticos.

\section{Incidência}

A incidência de gastroenterites agudas, nas crianças estudadas, foi de 2,78 episódios por 100 crianças/mês, com intervalo de confiança (95\%) entre 1,89 a 3,14 episódios por 100 crianças/mês (Tabela 2 ).

Nem todas as crianças apresentaram episódios de gastroenterites durante os 12 meses de seguimento. No subdistrito de paz da Barra Funda e nos bairros de Vila Ramos e Vila Penteado, 20\% das crianças apresentaram gastroenterites, enquanto que, em Itaberaba e no Parque Tietê, a proporção de crianças atingidas foi de $31 \%$. 
Tabela 2 - Episódios por criança afetada e incidência estimada de diarréias, por bairro, 1986 - 1987, Município de São Paulo.

Table 2 - Episodes of diarrhea by affected children and estimated incidence of diarrhea, by neighborhood, 19861987, city of S. Paulo.

\begin{tabular}{lcc}
\hline Bairros & $\begin{array}{c}\text { Episódios por } \\
\text { criança/ano } \\
\text { afetada }\end{array}$ & $\begin{array}{c}\text { Incidência* } \\
\text { por } 100 \text { crianças/mês } \\
\text { (IC) }\end{array}$ \\
\hline Barra Funda & 1,13 & $1,06-2,62$ \\
Vila Ramos & 1,15 & $1,32-3,06$ \\
Vila Penteado & 1,52 & $2,03-3,67$ \\
Parque Tietê & 1,58 & $3,33-5,53$ \\
Itaberaba & 1,92 & $6,07-6,53$ \\
\hline Total & 1,40 & $1,89-3,14$ \\
\hline * p $<0,01$ & \\
IC: Intervalo de confiança &
\end{tabular}

Tabela 3 - Incidência de gastroenterites agudas segundo idade, 1986-1987, M unicípio de São Paulo.

Table $\mathbf{3}$ - Incidence of acute gastroenteritis by age, 1986-1987, city of S. Paulo.

\begin{tabular}{lc}
\hline $\begin{array}{l}\text { Idade } \\
\text { (ano) }\end{array}$ & $\begin{array}{c}\text { Incidência por } \\
100 \text { crianças/mês }\end{array}$ \\
\hline$<1$ & 5,62 \\
1 & 4,11 \\
2 & 1,80 \\
3 & 3,15 \\
4 & 2,16 \\
5 & 2,80 \\
\hline Total & 2,78 \\
\hline
\end{tabular}

O número de episódios de gastroenterites por criança/ano afetada, em cada bairro, variou de 1,13 a 1,9 episódios por criança/ano (Tabela 2). Para as gastroenterites agudas a menor incidência ocorreu no subdistrito da Barra Funda e a maior, no bairro de Itaberaba, com diferencial de 3 vezes.

Os resultados apresentados na Tabela 3 mostram uma incidência mais alta em menores de um ano e nas crianças de um ano de idade com declínio progressivo nas demais faixas etárias.

A distribuição mensal das gastroenterites agudas apresentou maiores incidências entre julho e agosto e as menores em janeiro e fevereiro (Figura). A variação sazonal só foi estatisticamente significativa no Parque Tietê com incidências altas em agosto, setembro, outubro e janeiro. Nos demais bairros, analisados separadamente, as diferenças não se mostraram significantes.

As associações de possíveis fatores de risco com a incidência de gastroenterites entre as crianças estudadas constam na Tabela 4. Entre os fatores que apresentam associação temos que a incidência foi inversamente proporcional aos níveis de renda "per capita". As crianças cujas famílias tinham renda "per capita" inferior a um salário-mínimo, apresentaram

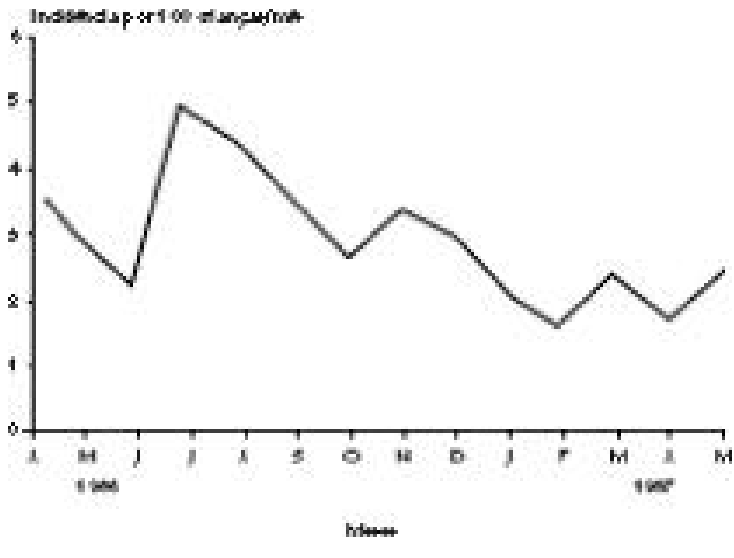

Figura - Distribuição sazonal das gastroenterites (Incidência por 100/crianças/mês). M unicípio de São Paulo, 1986-1987. Figure - Seasonal distribution of gastroenteritis (incidence by 100/children/month, city of S. Paulo, 1986-1987.

Tabela 4 - Fatores socioeconômicos e ambientais associados com a incidência das diarréias, 1986-1987, Município de São Paulo.

Table 4 - Socioeconomic and environment factors associated with the incidence of diarrhea, 1986-1987, city of S. Paulo.

\begin{tabular}{lcc}
\hline Fator & RR(bruto) & RR (ajustado) \\
\hline Renda “per capita" & 2,15 & $\mathrm{NS}$ \\
Tempo de fixação em S.Paulo & 1,38 & 1,38 \\
Saneamento básico & 4,50 & $\mathrm{NS}$ \\
Tratamento domiciliar da água & 4,30 & 4,48 \\
Condições de habitação: & & \\
a) Inadequada & 1,90 & $\mathrm{NS}$ \\
b) Precária & 2,50 & 2,50 \\
Diarréias freqüentes & 3,60 & 5,69 \\
Ausência de pré-natal & 3,20 & 9,75 \\
Parto prematuro & 2,30 & $\mathrm{NS}$ \\
Baixo peso ao nascer & $\mathrm{NS}$ & $\mathrm{NS}$ \\
Renda familiar & $\mathrm{NS}$ & $\mathrm{NS}$ \\
Amamentação e diarréias em & $\mathrm{NS}$ & $\mathrm{NS}$ \\
< de 2 anos & & \\
Aglomeração & 1,39 & $\mathrm{NS}$ \\
(5 pessoas ou + p/ dormitório) & & \\
Desnutrição & 1,34 & $\mathrm{NS}$ \\
Alimentação inadequada & $\mathrm{NS}$ & $\mathrm{NS}$ \\
\hline
\end{tabular}

NS : Não significante

$P<0,01$

RR-Risco relativo

incidência mais elevada de gastroenterites com risco relativo $(\mathrm{RR})$ de 2,15 em relação àquelas que não apresentavam este atributo.

A procedência dos pais e o tempo de fixação das famílias no município não contribuíram para diferenciais na incidência do agravo estudado. As crianças cujos domicílios não dispunham de instalações sanitárias e água encanada apresentaram alta incidência de gastroenterites (12,82 episódios/100 crianças/ano) com RR de 4,5 (p < 0,01). A ausência de tratamento domiciliar da água utilizada para beber e cozinhar (cloração, fervura e filtração) também es- 
teve associada à incidência mais elevada de gastroenterites; as crianças submetidas a essas condições apresentaram 11,9 episódios por 100 crianças/ano, com RR de 4,3 ( $\mathrm{p}<0,01)$.

As condições de habitação também se mostraram importantes, havendo gradiente inversamente proporcional entre a incidência de gastroenterites agudas e as condições adequadas de moradia . Nesse caso, o RR foi de 2,5 para crianças vivendo em habitações precárias $(\mathrm{p}<0,01)$ em relação às que viviam em condições adequadas.

Quando as mães referiram ocorrência freqüente de diarréias entre os antecedentes gastrointestinais das crianças, essas apresentaram incidência elevada de gastroenterites (7,92 episódios/100 crianças/ano) com RR de 3,6 em relação àquelas que não apresentam esse atributo ( $p<0,01)$. Já as crianças cujas mães relataram verminoses freqüentes, apresentaram incidência de gastroenterites semelhante à das crianças sem esses antecedentes.

As crianças cujas mães fizeram pré-natal e as gestações chegaram a termo, apresentaram incidências semelhantes independentemente do tipo de parto. Aquelas nascidas de parto prematuro apresentaram incidência mais alta de gastroenterites, com RR de $2,3(p<0,01)$ em relação às nascidas de parto a termo, e as crianças cujas mães não fizeram pré-natal, nascidas de gestação a termo e de parto cesareano, tiveram a mais alta incidência $(8,33$ episódios $/ 100$ crianças/ano), com RR de 3,2 ( $\mathrm{p}<0,01$ ) em relação às que fizeram pré-natal.

\section{ISCU SSÃO}

A incidência de diarréia encontrada na amostra estudada é aproximadamente 10 vezes menor do que a mediana observada em 276 inquéritos realizados em países subdesenvolvidos, no período de 19811986 e revisadas por Martines e col. ${ }^{19}$, e inferior àquelas encontradas, no início da década de 80 , na zona rural de Fortaleza, 11,9 episódios por 100 crianças $/$ mês $^{13}$, e em população de baixa renda na periferia de Manaus, 40 episódios por 100 crianças/mês ${ }^{12}$. A incidência encontrada no presente trabalho foi também 2,7 vezes menor do que a amostra de crianças, da mesma faixa etária, residentes no Município de São Paulo, estudada por Benício e col. ${ }^{5}$ em 1984/85.

Tais diferenças podem ser atribuídas às características das crianças estudadas, e à metodologia utilizada, às condições de vida das 5 áreas incluídas na presente pesquisa e à evolução das condições de saúde do Município de São Paulo, na década de 80, que lhe conferem atributos muito distintos das regiões do terceiro mundo onde foram desenvolvidos a maior parte dos estudos citados.

Entre as características do grupo estudado que devem ser levadas em conta, estão: a sub-representação dos menores de um ano; a inclusão de crianças com 5 anos completos, a inexistência de famílias residentes em favelas, às condições habitacionais (somente 3\% das famílias viviam em barracos), $\mathrm{o}$ acesso pela quase totalidade dos domicílios estudados a serviços de água e esgoto e ao nível socioeconômico das famílias que era superior à média da amostra das famílias residentes no Município de São Paulo, incluída em pesquisa realizada em $1984 / 85^{20}$. Por sua vez, dois dos três subdistritos de paz onde residiam as crianças estudadas, Barra Funda e Nossa Senhora do Ó, apresentam indicadores que sugerem condições de vida melhores do que o Município de São Paulo como um todo.

A análise dos indicadores demográficos, sociais e de infra-estrutura urbana, relativos ao Município de São Paulo, mostra uma evolução favorável que deve ter influenciado as modificações dos padrões de morbi-mortalidade por diarréias no Município, a partir da década de 80, com repercussões na mortalidade infantil. Entre esses indicadores cabe destacar o aumento da cobertura do saneamento básico e dos serviços de saúde, a alta cobertura de vacinação, especialmente contra o sarampo, a ampliação do acesso à escola e a diminuição do ritmo de crescimento demográfico, esse último determinado em boa parte pela diminuição das correntes migratórias.

Kolsky $^{17}$, em ampla análise da bibliografia disponível referente ao efeito de diversas medidas de intervenção na queda da morbi-mortalidade por diarréias, salienta que o impacto do saneamento básico como medida de intervenção para o controle das diarréias pode variar bastante de lugar para lugar, dependendo de fatores, tais como o grau de contaminação ambiental por fezes e o comportamento da população quanto a hábitos de higiene e seu nível de desenvolvimento socioeconômico.

Por outro lado, Murray e $\mathrm{Chen}^{23}$, analisando recentes teorias explicativas da tendência secular de declínio da mortalidade nos países do terceiro mundo, em boa parte influenciada pelo comportamento das gastroenterites na infância, tende a valorizar o efeito cumulativo de investimentos articulados, de longo prazo, no setor social, como a melhoria do nível de educação da mãe, da infra-estrutura dos serviços de saúde e da infra-estrutura urbana, conferindo menor importância à introdução isolada de tecnologias por meio da implementação de programas ou campanhas sanitárias. 
Quanto aos aspectos metodológicos do presente estudo que podem ter influenciado os resultados, talvez subestimando a incidência das diarréias, um deles é a definição de caso adotada. Essa questão é polêmica pois a síndrome diarréica não constitui uma única entidade nosológica podendo estar associada a inúmeras etiologias e seu diagnóstico fundamentase em alterações na consistência e na freqüência da eliminação das fezes que, por sua vez, pode variar em diferentes faixas etárias e populações conforme sua cultura. Essas variações explicam de certa forma a heterogeneidade das metodologias e definições de caso em diferentes estudos epidemiológicos a respeito das diarréias ${ }^{1}$. Um dos pontos da definição de caso que influencia substancialmente a comparabilidade de diferentes estudos é o intervalo de dias assintomáticos entre dois episódios. Simulações feitas com intervalos variando entre 1 e 7 dias mostraram que na medida em que aumenta o intervalo diminui a incidência das diarréias e superestima os casos de diarréia "persistentes", ou seja, com duração maior do que 14 dias, ainda que apresente pequeno efeito sobre a duração média dos $\operatorname{casos}^{1,22}$.

A duração média dos episódios de diarréias observados no presente estudo, a baixa taxa de hospitalização, a terapêutica utilizada e a ausência de óbitos refletem a pequena gravidade da doença. Esse resultado é coerente com a expressiva diminuição das internações determinadas por casos de desidratação, cerca de 50\%*, no Município de São Paulo, entre 1980 e 1985. Por sua vez, os 9,3\% de casos de diarréia "persistente", ou seja, com duração superior a 14 dias, aceito como marcador de risco para casos mais graves ${ }^{25}$, possivelmente encontram-se superestimado pela definição de caso adotada, que estabelece um intervalo de 7 dias assintomáticos entre dois episódios de diarréia ${ }^{1,22}$.

Por outro lado, diferentes agentes etiológicos devem igualmente influenciar a duração média dos episódios de diarréia ${ }^{6,15,26}$. Black e col. ${ }^{7}$, em Bangladesh, encontrou uma duração média de 4 a 5 dias para o conjunto de episódios, elevando-se para 7 dias as diarréias por shiguela e 9,5 dias para as diarréias por rotavírus em menores de 1 ano. Outro fator relacionado à duração dos episódios é o nível sócioeconô- mico. Guerrant ${ }^{13}$, em pesquisa realizada em localidade rural próxima de Fortaleza, verificou que crianças com melhores condições de vida apresentaram episódios com duração média de 4,2 dias, enquanto aquelas em condições mais desfavoráveis tiveram episódios com duração média de 31,3 dias.

$\mathrm{Na}$ medida em que não se dispõem de exames etiológicos para elucidação desses episódios, é difícil aquilatar o acerto ou não das terapêuticas empregadas. Tendo em vista, porém, a baixa taxa de hospitalização e o pequeno número de reidratações endovenosas, pode-se supor um excesso na utilização de antibióticos embora a proporção encontrada não tenha sido muito alta. Por sua vez, a utilização da hidratação oral em $36,7 \%$ dos casos sugere uma razoável divulgação deste procedimento entre as mães.

A variação da incidência de gastroenterites agudas com a idade atingindo principalmente os menores de 2 anos é compatível com a literatura ${ }^{19,27}$. A sazonalidade das diarréias verificada no presente estudo coincide com aquela descrita para os países desenvolvidos, onde as gastroenterites, principalmente as de etiologia viral, predominam no inverno, enquanto que, nos países em desenvolvimento observa-se incidência praticamente constante durante o ano todo $^{24,26}$. Segundo Robins-Browne ${ }^{24}$, as mudanças na sazonalidade das diarréias são acompanhadas de pronunciada diminuição da mortalidade associada a esse quadro mórbido, refletindo também a mudança do agente etiológico predominante, possivelmente a substituição da $E$. coli pelo rotavírus.

Os resultados da presente pesquisa apontam incidência não muito elevada das diarréias na população estudada, se comparada com dados disponíveis de regiões subdesenvolvidas. No entanto, permite identificar segmentos da comunidade mais vulneráveis a esse agravo, especialmente aqueles sem acesso a serviços de saúde e vivendo em habitações precárias desprovidas de saneamento básico. Entretanto, uma caraterística comum aos episódios identificados foi o caráter benigno desses eventos, permitindo que a maioria dos casos pudesse ser atendida em serviços de assistência primária apresentando boa evolução. 


\section{REFERÊN CIAS BIBLIO GRÁFICAS}

1. BAQUI, A.H. et al. Methodological issues in diarrhoeal diseases epidemiology: definition of diarrhoeal episodes. Int. J. Epidemiol., 20 : 1057-63, 1991.

2. BALDACCI, E.R. et al. Etiologia viral de casos de gastroenterite infantil: uma caracterização clínica. Rev.Saúde Pública, 13: 47-53, 1979.

3. BARATA, R.C.B. et al. Gastroentrites e infecções respiratórias agudas em crianças menores de 5 anos, em área da região Sudeste do Brasil, 1986-1987. I - Infecções respiratórias agudas. Rev. Saúde Pública, 30: 553-63, 1996.

4. BARRETO, M.L. et al. Mudanças dos padrões de morbimortalidade: uma revisão crítica das abordagens epidemiológicas. Physis , 3: 127-46,1993.

5. BENÍCIO, M.H.D’A. et al. Estudo das condições de vida das crianças do Município de São Paulo,SP(Brasil), 1984-1985.IV - Doença diarréica. Rev. Saúde Pública, 21: 23-8, 1987.

6. BLACK, R.E. et al. Enterotoxigenic Escherichia coli diarrhea: acquired immunity and transmission in an endemic area. Bull. World Health Organ., 59: 263-8, 1981.

7. BLACK, R.E. et al. Longitudinal studies of infectious diseases and physical growth of children in rural Bangladesh. Am. J. Epidemiol., 115: 315-24, 1982.

8. CARVALHEIRO, J.R. Levantamento de condições de saúde por entrevistas domiciliares. Ribeirão Preto, 1975. [Tese de Livre Docência - Faculdade de Medicina de Ribeirão Preto da USP].

9. FAYRAN, S.L. et al. Rotavirus gastroenterites in Southern California. Diag. Microbial. Infect. Dis., 7: 59-62, 1987.

10. FREBES, O. et al. Estudio de las gastroenterites en el Hospital Central de Valencia 1979-83. Isabelica Méd., 10: 139-67, 1985.

11. FUNDO DAS NAÇÕES UNIDAS PARA A INFÂNCIA (UNICEF ). Situação mundial da infância - 1995. Brasília, DF, 1995.

12. GIUGLIANO, L.G. et al. Longitudinal study of diarrhoeal incidence in a peri-urban community in Manaus (AmazonBrazil). Ann. Trop. Med. Parasitol., 80: 443-50,1986.

13. GUERRANT, R.L.et al. Prospective study of diarrhoeal disease in Northeastern Brazil: patterns of disease nutritional impact, etiologies and risk factors. J. Infect. Dis., 148: 98697, 1983.

14. GUERRANT, R.L. Twelve messages from enteric infections for science and society. Am. J. Trop. Med. Hyg., 51: 26-35,1994.
15. GURWITH, M. et al. A prospective study of rotavirus infection in infants and young children. J. Infec. Dis., 144: 218-24, 1981.

16. HJELT, K. et al. Acute gastroenterites in children attending day-care centres with special reference to rotavirus infection. Acta Paediatr. Scand., 76: 754-62, 1987.

17. KOLSKY, P.J. Diarrhoeal disease: current concepts and future challanges. Water, sanitation and dirrhoea: the limits of understaning. Trans. R. Trop. Med. Hyg., 87: 43-6, 1993.

18. LINHARES, A.C.et al. Prevalência de anticorpos para rotavirus em crianças diarréicas, Belém, Brasil. Rev. Fund. SESP, 28: 950-1005, 1983.

19. MARTINES, J. et al. Diarrheal diseases. In:Jamison, D.T. et al. Disease control priorities in developing countries. Oxford, Oxford University Press., 1993. p. 91-116 .

20. MONTEIRO, C.A. Saúde e nutrição das crianças de São Paulo: diagnóstico, contrastes sociais e tendências. São Paulo/Hucitec Edusp, 1988.

21. MONTEIRO, C.A. \& BENÍCIO, M.H.D. Determinants of infant mortality trends in developing countries - some evidence from S. Paulo city. Trans. R. Trop. Med. Hyg., 83: 5-9,1989.

22. MORRIS, S.S. et al. Diarrhoea: defining the episode. Int. J. Epidemiol., 23: 617-23, 1994.

23. MURRAY, J.L. \& CHEN, L.C. In search of a contemporary theory for understanding mortality change. Soc. Sci.Med., 36: 143-55, 1993.

24. ROBINS-BROWNE, R.M. Seasonal and racial incidence of infantile gastroenteritis in South Africa. Am.J.Epidemiol., 119: $350-5,1984$.

25. SAZAWAL, S. et al. Evidence for recent diarrhoeal morbidity as a risk fator for persistent diarrhoea : a casecontrol study. Int. J. Epidemiol., 20: 540-5,1991.

26. SENTURIA, Y.D. Epidemiology of rotavirus gastroenterites. J. Epidemiol. Community Health, 40: 236-9, 1986.

27. SNYDER, J.D. \& MERSON, M.H. The magnitude of the global problem of acute diarrhoeal disease: a review of active surveillance data. Bull. World Health Organ., 60: 605-13, 1982.

28. VICTORA, C. G. et al. Epidemiologia da desigualdade. São Paulo, Hucitec, 1988. 